\title{
Crystal structure of an RNA/DNA strand exchange junction
}

4 Joshua C. Cofsky ${ }^{1}$, Gavin J. Knott ${ }^{2}$, Christine L. Gee ${ }^{1,3,4}$, and Jennifer A. Doudna*1,3-8

$6{ }^{1}$ Department of Molecular and Cell Biology, University of California, Berkeley, Berkeley,

7 California, USA.

8 2Monash Biomedicine Discovery Institute, Department of Biochemistry \& Molecular

9 Biology, Monash University, VIC 3800, Australia.

$10{ }^{3}$ California Institute for Quantitative Biosciences (QB3), University of California,

11 Berkeley, Berkeley, California, USA.

$12{ }^{4}$ Howard Hughes Medical Institute, University of California, Berkeley, Berkeley,

13 California, USA.

$14{ }^{5}$ Department of Chemistry, University of California, Berkeley, Berkeley, California, USA.

$15{ }^{6}$ Molecular Biophysics and Integrated Bioimaging Division, Lawrence Berkeley National

16 Laboratory, Berkeley, Berkeley, California, USA.

$17{ }^{7}$ Innovative Genomics Institute, University of California, Berkeley, Berkeley, California,

18 USA.

$19{ }^{8}$ Gladstone Institutes, University of California, San Francisco, San Francisco, California, 20 USA.

$22{ }^{*}$ Corresponding author

23 E-mail: doudna@berkeley.edu (J.A.D.) 


\section{Abstract}

25 Short segments of RNA displace one strand of a DNA duplex during diverse processes

26 including transcription and CRISPR-mediated immunity and genome editing. These

27 strand exchange events involve the intersection of two geometrically distinct helix

28 types-an RNA:DNA hybrid (A-form) and a DNA:DNA homoduplex (B-form). Although

29 previous evidence suggests that these two helices can stack on each other, it is

30 unknown what local geometric adjustments could enable A-on-B stacking. Here we

31 report the X-ray crystal structure of an RNA-5'/DNA-3' strand exchange junction at an

32 anisotropic resolution of 1.6 to $2.2 \AA$. The structure reveals that the A-to-B helical

33 transition involves a combination of helical axis misalignment, helical axis tilting and

34 compression of the DNA strand within the RNA:DNA helix, where nucleotides exhibit a

35 mixture of A- and B-form geometry. These structural principles explain previous

36 observations of conformational stability in RNA/DNA exchange junctions, enabling a

37 nucleic acid architecture that is repeatedly populated during biological strand exchange

38 events. 


\section{Introduction}

41 Although structural and mechanistic information is available for various types of DNA

42 strand exchange processes [1-8], comparatively little is known about RNA/DNA strand

43 exchange. In this reversible process, a strand of RNA hybridizes to one strand of a DNA

44 duplex while displacing the other strand, requiring concomitant disruption of DNA:DNA

45 base pairs and formation of RNA:DNA base pairs. This process occurs most notably at

46 the boundaries of R-loops, such as those left by transcriptional machinery [9], those

47 employed by certain transposons $[10,11]$, or those created by CRISPR-Cas (clustered

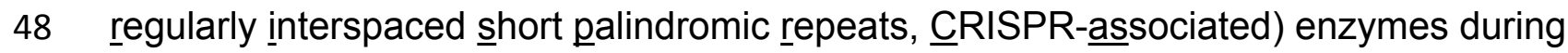

49 prokaryotic immunity or eukaryotic genome editing [12-15]. Structural insight into

50 RNA/DNA strand exchange could therefore improve our understanding of how

51 transcriptional R-loops are resolved and how CRISPR-Cas enzymes such as Cas9

52 manipulate R-loops to efficiently reject off-target DNA and recognize on-target DNA.

The defining feature of RNA/DNA strand exchange is the junction where the

54 RNA:DNA helix abuts the DNA:DNA helix. Previous experiments on exchange junctions

55 containing an RNA-5' end and a DNA-3' end (an "RNA-5'/DNA-3' junction," which is the

56 polarity generated by Cas9) showed the component DNA:DNA duplex to be more

57 thermodynamically stable than a free DNA helix end, perhaps due to interhelical

58 RNA:DNA/DNA:DNA stacking [16]. While stacking in DNA-only junctions is thought to

59 occur as it would in an uninterrupted B-form duplex $[8,17,18]$, an analogous structural

60 prediction cannot be made for RNA/DNA junctions because the two component helices

61 are predisposed to different geometries: B-form for the DNA:DNA helix and a variant of

62 A-form for the RNA:DNA helix [19-21]. A conformation that preserves base stacking 
63 across such a junction must reconcile base pairs that are flat and centered (B-form) with

64 base pairs that are inclined and displaced from the helical axis (A-form). While prior

65 structural studies of Okazaki fragments reckoned with a similar geometric puzzle [22],

66 Okazaki fragments bear an RNA-3'/DNA-5' polarity (opposite of the polarity addressed

67 here) and lack the strand discontinuity that defines exchange junctions. Thus, the

68 structural basis for the putative stacking-based stability in RNA-5'/DNA-3' junctions

69 remains unknown.

71 exchange junction, which undergoes an A-to-B transition without loss of base pairing or

72 stacking across the exchange point. This structure reveals the principles of global

73 helical positioning and local adjustments in nucleotide conformation that allow

74 RNA:DNA duplexes to stack on DNA:DNA duplexes in the RNA-5'/DNA-3' polarity. This

75 model also complements previously determined cryo-electron microscopy structures of

76 DNA-bound Cas9 for which poor local resolution in the original maps prevented

77 accurate modeling of the leading R-loop edge.

\section{Results}

80 Inspired by previous crystallographic studies of double-stranded DNA dodecamers

$81[23,24]$, we designed crystallization constructs that contained a "template" DNA strand

82 (12 nucleotides) and two "exchanging" RNA and DNA oligonucleotides that were

83 complementary to each half of the template DNA strand. In different versions of these

84 constructs, we varied the polarity (RNA-5'/DNA-3' vs. RNA-3'/DNA-5') and the internal

85 termini, which were either flush (exchanging oligonucleotides were 6-mers) or extended 
86 with a one-nucleotide flap that was not complementary to the template strand

87 (exchanging oligonucleotides were 7-mers, "flapped"). Only the flapped construct in the RNA-5'/DNA-3' polarity (Fig 1A) yielded well-diffracting crystals (anisotropic resolution of

891.6 to $2.2 \AA$ ). Thus, all results discussed here describe a flapped RNA-5'/DNA-3' strand exchange junction, which is the polarity previously observed to stabilize the component

91 DNA:DNA duplex [16].

Fig 1. Stabilizing features of the crystal lattice.

(A) Crystallization construct sequence. Black, DNA; red, RNA. (B) Schematized drawing (not to scale) of the crystal lattice along a direction that depicts the helical network formed by Molecules 1 and 2. Green shading, Molecule 1; blue shading, Molecule 2; orange shading, Molecule 3 (cross section). (C) Similar to panel B, but along a direction that depicts the helical network formed by Molecule 3. (D) Asymmetric unit colored by atomic B-factor. The thickness of the cartoon model also reflects the local B-factors. (E) Model and $2 \mathrm{mF}_{\mathrm{o}}-\mathrm{DF} \mathrm{F}_{\mathrm{c}}$ map (sharpened by $-38 \AA^{2}$, displayed at $3.3 \sigma$ ) of the Ade-Ade and Cyt-Cyt base pairs (contributed by the flap nucleotides of Molecules 1 and 2) that bridge

102 the helical network formed by Molecule 3. Distortion in the map is due to diffraction 103 anisotropy (see Methods).

We determined the X-ray crystal structure of the exchange junction (Table 1). In

106 this structure, the asymmetric unit contains three molecules (a "molecule" comprises

107 one DNA 12-mer and its complementary RNA and DNA 7-mers). The crystal lattice is

108 largely stabilized by nucleobase stacking interactions both within and between 
109 molecules. Along one lattice direction, Molecules 1 and 2 form a continuous network of

110 stacked helices, in which the external RNA:DNA duplex terminus of each Molecule 1

111 stacks on the equivalent terminus of Molecule 2, with a similar reciprocal interaction for

112 the external DNA:DNA duplex termini (a "head-to-head" and "foot-to-foot" arrangement)

113 (Fig 1B). Along another lattice direction, symmetry-related instances of Molecule 3

114 create a head-to-foot helical network (Fig 1C). Compared to Molecules 1 and 2,

115 Molecule 3 is poorly ordered (Fig 1D), and its atomic coordinates appear less

116 constrained by the data due to diffraction anisotropy (see Methods). In the Molecule 3

117 helical network, two base pairs formed between the flapped nucleotides of Molecules 1

118 and 2 bridge the duplex ends. The bridging nucleotides form a type I adenine-adenine

119 (ribonucleotide) base pair and a type XV hemiprotonated cytosine-cytosine

120 (deoxyribonucleotide) base pair [25] (Fig 1C, E).

122 Table 1. Crystallographic data and refinement statistics

\begin{tabular}{|l|r|}
\hline & \multicolumn{2}{|l|}{$\begin{array}{l}\text { RNA-5'/DNA-3' strand exchange } \\
\text { junction (PDB XXXX) }\end{array}$} \\
\hline Data collection & 1.116 \\
\hline Wavelength $(\AA)$ & $35.335-1.637(1.781-1.637)$ \\
\hline Resolution range $(\AA)$ & 1.656 \\
\hline Diffraction limit \#1 $(\AA)$ & $0.8648,-0.0396,-0.5006$ \\
Principal axes (orthogonal basis) & $0.657 a^{*}-0.168 b^{*}-0.735 c^{*}$ \\
\hline Principal axes (reciprocal lattice) & $0.1677,0.9624,0.2136$ \\
\hline Diffraction limit \#2 $(\AA)$ & $0.152 a^{*}+0.981 b^{*}+0.117 c^{*}$ \\
\hline $\begin{array}{l}\text { Principal axes (orthogonal basis) } \\
\text { Principal axes (reciprocal lattice) }\end{array}$ & $0.4733,-0.2686,0.8389$ \\
\hline $\begin{array}{l}\text { Diffraction limit \#3 }(\AA) \\
\text { Principal axes (orthogonal basis) } \\
\text { Principal axes (reciprocal lattice) }\end{array}$ & \\
\hline Space group & $0.397 a^{*}-0.342 b^{*}+0.852 c^{*}$ \\
\hline Unit cell & \\
\hline a, b, c $(\AA)$ & $37.025,43.586,52.182$ \\
\hline
\end{tabular}




\begin{tabular}{|c|c|}
\hline$\alpha, \beta, \gamma\left(^{\circ}\right)$ & $92.06,103.72,99.95$ \\
\hline Total reflections & $147975(7830)$ \\
\hline Unique reflections & $24808(1240)$ \\
\hline Multiplicity & $6.0(6.3)$ \\
\hline Spherical completeness (\%) & $64.7(14.5)$ \\
\hline Ellipsoidal completeness (\%) & $86.8(50.0)$ \\
\hline$<\mid / \sigma(I)>$ & $15.6(1.5)$ \\
\hline \multicolumn{2}{|l|}{ Wilson B-factor $\left(\AA^{2}\right)$} \\
\hline Eigenvalue \#1 $(\AA)$ & 48.57 \\
\hline Principal axes (orthogonal basis) & $0.9603,-0.1659,-0.2243$ \\
\hline Principal axes (reciprocal lattice) & $0.799 a^{*}-0.323 b^{*}-0.507 c^{*}$ \\
\hline Eigenvalue \#2 $(\AA)$ & 86.73 \\
\hline Principal axes (orthogonal basis) & $0.2257,0.9345,0.2753$ \\
\hline Principal axes (reciprocal lattice) & $0.209 a^{*}+0.961 b^{*}+0.183 c^{*}$ \\
\hline Eigenvalue \#3 $(\AA)$ & 45.47 \\
\hline Principal axes (orthogonal basis) & $0.1639,-0.3150,0.9348$ \\
\hline Principal axes (reciprocal lattice) & $0.123 a^{*}-0.300 b^{*}+0.946 c^{*}$ \\
\hline$R_{\text {merge }}$ & $0.037(1.293)$ \\
\hline$R_{\text {meas }}$ & $0.041(1.410)$ \\
\hline $\mathrm{R}_{\mathrm{pim}}$ & $0.016(0.556)$ \\
\hline $\mathrm{CC}_{1 / 2}$ & $0.999(0.474)$ \\
\hline \multicolumn{2}{|l|}{ Refinement } \\
\hline Resolution range $(\AA)$ & $35.34-1.641(1.768-1.641)$ \\
\hline Reflections used in refinement & $24717(1054)$ \\
\hline Reflections used for $R_{\text {free }}$ & $1223(41)$ \\
\hline $\mathrm{R}_{\text {work }}$ & $0.2371(0.3692)$ \\
\hline$R_{\text {free }}$ & $0.2846(0.3561)$ \\
\hline $\mathrm{CC}_{\text {work }}$ & $0.912(0.616)$ \\
\hline $\mathrm{CC}_{\text {free }}$ & $0.939(0.562)$ \\
\hline Number of non-hydrogen atoms & 1651 \\
\hline macromolecules & 1584 \\
\hline ligands & 0 \\
\hline solvent & 67 \\
\hline Protein residues & 0 \\
\hline RMSD - bond lengths $(\AA)$ & 0.014 \\
\hline RMSD - angles $\left({ }^{\circ}\right)$ & 1.42 \\
\hline Clashscore & 0.00 \\
\hline Average B-factor & 59.75 \\
\hline macromolecules & 60.15 \\
\hline solvent & 50.35 \\
\hline Number of TLS groups & 15 \\
\hline
\end{tabular}

123 Diffraction limits and eigenvalues of overall anisotropy tensor on $|F| s$ are displayed

124 alongside the corresponding principal axes of the ellipsoid fitted to the diffraction cut-off

125 surface as direction cosines in the orthogonal basis (standard PDB convention), and in 
126 terms of reciprocal unit-cell vectors. Statistics for the highest-resolution shell are shown 127 in parentheses.

130 pairing at all twelve nucleotides of the template DNA strand, and they are generally

131 similar in conformation $\left(\mathrm{RMSD}_{\mathrm{Mol} 1, \mathrm{Mol} 2}=0.703 \AA ; \mathrm{RMSD}_{\mathrm{Mol} 1, \mathrm{Mol} 3}=1.528 \AA\right.$,

$132 \mathrm{RMSD}_{\mathrm{Mol} 2, \mathrm{Mol} 3}=1.770 \AA$ ) $($ Fig 2A). The most dramatic differences are between Molecules

$1331 / 2$ and Molecule 3. For example, Molecule 3's flapped nucleotides form no

134 intermolecular base pairs, and the conformation of the DNA flap is flipped relative to

135 Molecules 1/2. Additionally, the external three base pairs of Molecule 3's DNA:DNA

136 helix tilt slightly toward the major groove as compared to the equivalent positions of

137 Molecules 1/2. Notably, the similarity of all three molecules at the three base pairs on

138 either side of the exchange point $\left(\mathrm{RMSD}_{\mathrm{Mol} 1, \mathrm{Mol} 2}=0.567 \AA ; \mathrm{RMSD}_{\mathrm{Mol} 1, \mathrm{Mol} 3}=0.502 \AA\right.$,

$139 \mathrm{RMSD}_{\mathrm{Mol} 2, \mathrm{Mol} 3}=0.751 \AA$ ) suggests that the conformation in this region represents a low140 energy solution to the stacking of RNA:DNA and DNA:DNA helices.

142 Fig 2. Molecule-to-molecule similarity and hydrogen bonding at the flapped 143 nucleotides.

144 (A) All-atom alignment of the three molecules in the asymmetric unit. Green, Molecule 145 1; blue, Molecule 2; orange, Molecule 3. Molecules 2 and 3 were aligned to Molecule 1

146 in this depiction. (B) Hydrogen bonding at the flapped nucleotides of Molecule 1. Dotted

147 lines indicate hydrogen bonds, and adjacent numbers indicate interatomic distance in $\AA$.

148 Black, DNA; red, RNA. This hydrogen bonding pattern is also observed in Molecule 2

149 but not in Molecule 3. 
At the exchange point of Molecules 1 and 2, the flapped nucleotides are stabilized not only by intermolecular base pairing (Fig 1C, E) and intramolecular

153 stacking (Fig 2B), but also by hydrogen bonds between sugar hydroxyls and backbone 154 phosphates. Specifically, at the junction-proximal phosphodiester within the DNA:DNA 155 helix, the pro- $S_{\mathrm{p}}$ and pro- $R_{\mathrm{p}}$ oxygens are hydrogen-bonded to the terminal 3 ' hydroxyl of 156 the flapped DNA nucleotide and the terminal 5' hydroxyl of the flapped RNA nucleotide,

157 respectively. Additionally, the pro- $S_{p}$ oxygen of the flapped DNA nucleotide is hydrogen158 bonded to the 2' hydroxyl of the flapped RNA nucleotide (Fig 2B). If the flaps were 159 longer than one nucleotide, as would occur during biological strand exchange events, 160 the hydrogen bonds to the terminal $3^{\prime} / 5^{\prime}$ hydroxyls would be perturbed. However, in 161 Molecule 3, the flipped deoxycytidine conformation precludes all the mentioned 162 extrahelical hydrogen bonds, yet the base-paired nucleotides within the junction are 163 conformationally similar to the same region in Molecules 1 and 2 (Fig 2A). Therefore, 164 we expect that the structural features of interest to this work-that is, the conformation 165 of the base-paired nucleotides immediately adjacent to the junction—would be 166 populated by junctions bearing flush RNA/DNA ends or flaps of arbitrary length. To understand the nature of the transition in helical geometry across the junction, 168 we performed alignments of regularized A-form and B-form DNA:DNA helices with the 169 observed RNA:DNA and DNA:DNA helices, respectively. These alignments revealed 170 that the DNA:DNA helix closely approximates perfect B-form geometry, especially in the 171 nucleotides closest to the junction (Fig 3A-C). Likewise, the RNA strand of the

172 RNA:DNA helix closely approximates A-form geometry (Fig 3A-C). On the other hand, 
173 the DNA strand of the RNA:DNA helix deviates from its A-form trajectory in the three

174 nucleotides that approach the exchange point, where the backbone is compressed

175 toward the minor groove (Fig 3B, D).

176

177 Fig 3. Alignments to regularized A-form/B-form helices.

178 (A) Black, DNA of Molecule 1; red, RNA of Molecule 1; white, regularized B-form

179 DNA:DNA helix aligned to the $6 \mathrm{bp}$ of Molecule 1's DNA:DNA helix; pink, regularized A-

180 form DNA:DNA helix aligned to the 6 bp of Molecule 1's RNA:DNA helix. (B) Cartoon

181 depiction, focused on the continuous strand. The alignment procedure for each 6-bp

182 block was identical to that performed in panel A, but in this depiction, the B-form (white)

183 and A-form (pink) helices were extended by an additional 6 bp (extended nucleotides

184 were not considered during alignment) to illustrate the path that the helix would take if

185 continuing along a perfect B-form or A-form trajectory. EP, exchange point (that is, the

186 phosphodiester or gap lying between the two nucleotides where the helix changes from

187 RNA:DNA to DNA:DNA). (C) Similar to panel B, but focused on the discontinuous

188 strand. (D) Close-up of the same representation depicted in panel A, focused on the

189 nucleotides that deviate most dramatically from the aligned A-form helix.

Interestingly, calculation of $z_{P}$, a geometric parameter that differentiates A-form

192 from B-form base steps [26], indicated that the RNA:DNA base step adjacent to the

193 exchange point is A-like, while the base steps in the center of the RNA:DNA helix are

194 intermediate in their A/B character (Fig 4A). This result indicates an important distinction

195 between strand trajectory (in terms of global alignment to a regularized A-form or B-form 
196 helix) and the local nucleotide conformations that underlie the trajectory. In the

197 RNA:DNA helix, the departure from A-form trajectory observed at junction-adjacent

198 nucleotides appears to result from non-A conformations at more junction-distal

199 nucleotides. Other indicators of helical geometry also suggest a mixture of A and B

200 character across the RNA:DNA helix (S1 Fig).

201

202 Fig 4. Geometric details of the A-to-B transition.

203 (A) For a given base step, the parameter $z_{P}$ is the mean of the $z$-displacement of the 204 two phosphorus atoms from the dimer's reference $x y$-plane. Note that $z_{P}$ is defined by a 205 pair of dinucleotides, so there are only 11 data points for a 12-bp helix, and integral x206 values lie between the base pairs in the diagram. This parameter was originally 207 introduced for its utility in distinguishing A-form from B-form base steps. Black, DNA;

208 red, RNA. (B) $X$ and $\delta$ are the two nucleotide torsion angles that best distinguish A-form

209 from B-form geometry. Note that these torsion angles are defined for each individual

210 nucleotide, so there are 24 data points for a 12-bp helix. Integers in red refer to

211 individual nucleotides, as indicated in the schematic at the bottom. Dashed ellipses

212 were drawn to match those depicted in [27]. (C) Y-displacement. Similar to $z_{P}$, this

213 parameter describes base steps (pairs of dinucleotides), not individual nucleotides. This

214 parameter cannot distinguish A-form from B-form geometry. Instead, note that the base

215 step across the exchange point dramatically departs from both A-form and B-form 216 geometry. 
To probe helical geometry with strand specificity, we calculated $X$ and $\delta$, nucleotide torsion angles that differ in A-form vs. B-form helices [27]. These parameters revealed that the irregularities observed in the paired base step parameters (Fig 4A and

221 S1 Fig) arise entirely from the template DNA strand, which flips between A- and B-like

222 conformations within the RNA:DNA hybrid (Fig 4B and S2 Fig). In contrast, the RNA

223 strand is entirely A-like, and all nucleotides of the DNA:DNA helix are B-like except at

224 position 12 of the continuous strand, which is likely due to an end effect. These

225 observations agree with the conclusions drawn from the alignments (Fig 3A), and they

226 highlight the DNA strand of the RNA:DNA helix as the structure's most geometrically

227 irregular region, which may enable the junction-adjacent deviation in trajectory.

In addition to the distortions in the continuous DNA strand, the geometric switch

229 also seems to depend on the break in the discontinuous strand, which facilitates a

230 marked jump in the backbone trajectory across the exchange point (Fig $3 \mathrm{C}$ ). This

231 feature reflects a global jump in helical positioning that is visualized most clearly in the

232 aligned regularized A-form and B-form duplexes, whose helical axes are tilted and 233 misaligned with respect to each other (the helical axes are tilted from parallel by $14^{\circ}$,

$234 \mathrm{Mol}$; $18^{\circ}$, Mol2; $2^{\circ}$, Mol3) (Fig 2A and Fig 3B, C). Axis misalignment is detectable in the

235 large positive y-displacement value across the central base step, which deviates

236 dramatically from the expected value $(0 \AA)$ for either an A-form or B-form duplex (Fig

237 4C). This observation emphasizes the exchange point as a special base step with 238 noncanonical alignment, made possible by discontinuity in the exchanging strands. 
241 Together, our data suggest that stacking an RNA:DNA helix on a DNA:DNA helix does

242 not require deviation of the RNA strand or either strand of the DNA:DNA helix from their

243 native A-form or B-form conformations, respectively. Instead, continuous stacking

244 appears to result from a combination of three structural principles. First, alternating A-

245 like and B-like nucleotide conformations in the hybrid's DNA strand compress the strand

246 relative to a pure A-form trajectory (Fig 3B, D, Fig 4B, Fig 5A). Due to A-form base pair

247 inclination ( $20^{\circ}$ from perpendicular to the helical axis) in RNA:DNA duplexes, the DNA

248 naturally juts further along the helical axis than the RNA at the RNA-5' end. This slanted

249 RNA:DNA end can be stacked upon a flat DNA:DNA end through strand-specific

250 compression - that is, compression of the hybrid's protruding DNA strand (Fig 5A).

251 Second, an alternative to strand compression is to tilt the helical axes themselves,

252 which occurs in Molecules 1 and 2 but not Molecule 3 (Fig 2A and Fig 5A). Third, the

253 helical centers are misaligned at the exchange point (Fig 3B, C and Fig 4C), which

254 effectively aligns the off-center base pairs of the A-form duplex with the centered base

255 pairs of the B-form duplex (Fig 5B).

257 Fig 5. Structural principles of A-on-B stacking at the RNA-5'/DNA-3' strand

258 exchange junction.

259 (A) Simplified schematics illustrating strand-specific compression and tilting of the

260 helical axes. The slanted appearance of the RNA:DNA duplex is intended to represent

261 the base pair inclination characteristic of A-form duplexes, which pushes the 3' DNA end

262 farther along the helical axis than the 5' RNA end. Black, DNA; red, RNA. (B) Helical

263 cross-sections. Black, DNA:DNA helix; red, RNA:DNA helix. The rectangle represents 
264 the base pair nearest the exchange point (centered in the B-form helix, off-center in the

265 A-form helix). The solid circle represents the helical axis. The true stacking solution is a

266 combination of the three principles illustrated here, although Molecule 3 does not exhibit

267 tilting.

This new structure is best examined in the context of previous structural studies of RNA:DNA/DNA:DNA junctions emulating Okazaki fragments, which include a

271 chimeric (covalently continuous) RNA-DNA strand. When crystallized, these fragments

272 assumed an entirely A-form conformation, even within the DNA:DNA duplex [28-32].

273 However, in solution, Okazaki fragments resembled the present structure in that they

274 were A-like within the RNA:DNA helix and B-like within the DNA:DNA helix [22,33-36].

275 Solution structures also exhibited a tilt between the RNA:DNA/DNA:DNA helical axes

276 and intermediate nucleotide geometry within the DNA of the hybrid. Because

277 intermediate geometry is a known feature of the DNA of any RNA:DNA hybrid [19,20], it

278 may be the natural inclination of this more geometrically ambiguous strand to

279 accommodate the A-to-B transition as it does in the present structure. Notably, dramatic

280 misalignment of the RNA:DNA/DNA:DNA helical centers is observed only in the present

281 structure and is likely enabled by the break in the exchanging strands, which is not a

282 feature of Okazaki fragments.

Because stable stacking of another duplex on a DNA:DNA terminus is expected

284 to inhibit duplex melting [37], the structural principles illuminated here may explain the

285 rigidity that we previously observed in the DNA:DNA duplex of RNA-5'/DNA-3' exchange

286 junctions [16]. However, it is also possible that different sequences or environments 
287 promote different conformational preferences than those observed in this crystal

288 structure. Previously, we also observed that the DNA:DNA duplex in junctions of the

289 opposite polarity (RNA-3'/DNA-5') is destabilized relative to a non-exchanging terminus

290 [16]. Unfortunately, because that junction type failed to crystallize under our tested

291 conditions, this odd asymmetry in junction structure remains unexplained.

Nevertheless, the stacked RNA-5'/DNA-3' structure determined here represents

293 a key conformation that is likely populated throughout RNA/DNA exchange events,

294 including those mediated by the genome-editing protein Cas9. Branch migration is

295 crucial to Cas9 target search, which involves repeated R-loop formation (RNA invades a

296 DNA:DNA duplex) and resolution (DNA invades an RNA:DNA duplex) until the true

297 target is located [15]. During this process, the leading R-loop edge likely passes through

298 interhelically stacked states between base pair formation and breakage events.

299 Consistent with this prediction, in some cryo-electron microscopy structures depicting

300 Cas9-bound R-loops, the leading (RNA-5'/DNA-3') R-loop edge appeared interhelically

301 stacked $[38,39]$. While local resolution was insufficient to enable accurate atomic

302 modeling of the exchange junction from the original electron microscopy maps, our

303 high-resolution crystal structure provides a new geometric standard for modeling this

304 kind of junction.

306 grows or shrinks, stacking must be disrupted at the junction [8]. Thus, in addition to the

307 stacked structure determined here, which can be interpreted as a ground state, strand

308 exchange also requires passage through unstacked conformations, some of which may

309 resemble the junction structures seen in other Cas9-bound R-loops [40,41]. A complete 
310 model of RNA/DNA strand exchange, then, will rely on a structural and energetic

311 understanding of the junction in both stacked and unstacked states, and it will account

312 for the effects of the proteins acting in R-loop formation and resolution.

313 


\section{Methods}

\section{Oligonucleotide synthesis and sample preparation}

316 All oligonucleotides (DNA 12-mer $\left\{5^{\prime}-\right.$ GTAAGCAGCATC-3'\}; DNA 7-mer \{5'-GATGCTC-

$3173\}$; RNA 7-mer $\left.\left\{5^{\prime}-A G C U U A C-3\right\}\right)$ were synthesized and purified by Integrated DNA

318 Technologies (high-performance liquid chromatography (HPLC) purification for DNA

319 oligonucleotides and RNase-free HPLC purification for the RNA oligonucleotide). Dry

320 oligonucleotides were dissolved in nuclease-free water (Qiagen), and concentrations

321 were estimated by Nanodrop (Thermo Scientific) absorbance measurements with

322 extinction coefficients estimated according to [42] (DNA 12-mer, $\varepsilon_{260}=135200 \mathrm{M}^{-1} \cdot \mathrm{cm}^{-1}$;

323 DNA 7-mer, $\varepsilon_{260}=70740 \mathrm{M}^{-1} \cdot \mathrm{cm}^{-1}$; RNA 7-mer, $\left.\varepsilon_{260}=75580 \mathrm{M}^{-1} \cdot \mathrm{cm}^{-1}\right)$. The three

324 oligonucleotides were combined and diluted in water, each at $500 \mu \mathrm{M}$ final

325 concentration. This exchange junction sample was incubated at $50^{\circ} \mathrm{C}$ for 10 minutes,

326 cooled to $25^{\circ} \mathrm{C}$ within a few seconds, and used directly in the crystallization setups

327 described below.

\section{Crystallization and data collection}

330 Initial screens were performed using Nucleix and Protein Complex suites (Qiagen) in a

331 sitting-drop setup, with $200 \mathrm{~nL}$ of sample added to $200 \mathrm{~nL}$ of reservoir solution by a

332 Mosquito instrument (SPT Labtech) and incubated at either $4^{\circ} \mathrm{C}$ or $20^{\circ} \mathrm{C}$. Several

333 conditions yielded crystals within one day, and initial hits were further optimized at a

334 larger scale. The crystal used for the final dataset was produced as follows: $0.5 \mu \mathrm{L}$ of

335 sample was combined with $0.5 \mu \mathrm{L}$ reservoir solution $(0.05 \mathrm{M}$ sodium succinate $(\mathrm{pH} 5.3)$, 
$3360.5 \mathrm{mM}$ spermine, $20 \mathrm{mM}$ magnesium chloride, 2.6 M ammonium sulfate) in a hanging-

337 drop setup over $500 \mu \mathrm{L}$ reservoir solution, and the tray was stored at $20^{\circ} \mathrm{C}$. Crystals

338 formed within one day and remained stable for the 2.5 weeks between tray setting and

339 crystal freezing. A crystal was looped, submerged in cryoprotection solution (0.05 M

340 sodium succinate ( $\mathrm{pH}$ 5.3), $0.5 \mathrm{mM}$ spermine, $20 \mathrm{mM}$ magnesium chloride, $3 \mathrm{M}$

341 ammonium sulfate) for a few seconds, and frozen in liquid nitrogen. Diffraction data

342 were collected under cryogenic conditions at the Advanced Light Source beamline 8.3.1

343 on a Pilatus3 S 6M (Dectris) detector.

Data processing, phase determination, and model refinement

346 Preliminary processing of diffraction images was performed in XDS [43,44]. Unmerged

347 reflections underwent anisotropic truncation, merging, and anisotropic correction using

348 the default parameters of the STARANISO server (v3.339) [45], and a preliminary

349 structural model was included in the input to estimate the expected intensity profile. The

350 best-fit cut-off ellipsoid imposed diffraction limits of $1.656 \AA$, 2.176 $\AA$, and $1.637 \AA$ based

351 on a cut-off criterion of $\mathrm{I} / \sigma(\mathrm{I})=1.2$. The "aniso-merged" output MTZ file was used for

352 downstream processing. Using programs within CCP4 (v7.1.015), $\mathrm{R}_{\mathrm{free}}$ flags were

353 added to $5 \%$ of the reflections, and reflections outside the diffraction cut-off surface

354 were removed.

355 Phases were determined by molecular replacement with Phaser-MR [46], as

356 implemented in Phenix v1.19.2-4158 [47]. The search model comprised two

357 components (unconstrained with respect to each other), both generated in X3DNA v2.4

358 [48] and each representing one half of the base-paired portion of the crystallization 
construct. The first component was a 6-base-pair RNA:DNA duplex with perfect A-form geometry and sequence 5'-GCUUAC-3' / 5'-GTAAGC-3' (created using the program "fiber" with the -rna option, followed by manual alteration of the DNA strand in PyMOL

v2.4.1). The second component was a 6-base-pair DNA:DNA duplex with perfect B-form geometry and sequence 5'-GATGCT-3' / 5'-AGCATC-3' (created with "fiber" option -4).

364 Successful phasing was achieved by searching for three copies of each of these components (six components total). Additional phosphodiesters and nucleotides were built in Coot v0.9.2 [49], and the model underwent iterative refinements in Phenix.

367 Phasing and preliminary refinements were initially performed using an earlier (lowerresolution) dataset that had similar unit cell parameters to the final dataset described

369 above.

The initial model, which was refined into a map generated from the earlier

371 dataset, was rigid-body docked into the final-dataset-derived map and underwent further

372 iterative refinements, beginning with resetting of the atomic B-factors, simulated

373 annealing, and addition of ordered solvent. Non-crystallographic symmetry restraints

374 were applied in early rounds of refinement to link the torsion angles of the three

375 molecules within the asymmetric unit; these restraints were removed in the final rounds

376 of refinement. TLSMD [50,51] was used to determine optimal segmentation for

377 Translation/Libration/Screw (TLS) refinement (each 7-mer comprised a separate

378 segment, and the 12-mers were each divided into three segments: nucleotides 1-4, 5-8,

379 9-12). Refinement using Phenix's default geometry library yielded dozens of bond

380 lengths and angles that were marked as outliers by the PDB validation server, so the

381 faulty parameters were rigidified ad hoc (that is, their estimated standard deviation 
382 values in the library files were made smaller, with no change to the mean values). The

383 final three cycles of refinement were performed in Phenix with adjustments to XYZ

384 (reciprocal-space), TLS (segments as indicated above), and individual B-factors. In

385 Table 1, STARANISO and Phenix were used to calculate the data collection statistics

386 and the refinement statistics, respectively.

The final $R_{\text {free }}$ value $(0.283)$ is higher than expected for a structure refined using diffraction data at a resolution of $1.6 \AA$ [52]. However, it is important to note that the highest-resolution shell has a completeness of just $6 \%$, and completeness only rises above $95 \%$ at $\sim 2.3 \AA$, due mostly to the anisotropic nature of the diffraction data.

Additionally, due to diffraction anisotropy, the $2 \mathrm{mF}_{\mathrm{o}}-\mathrm{DF}_{\mathrm{c}}$ map appears distorted along certain dimensions, affecting interpretation of Molecule 3 most negatively. Therefore, the geometric details of Molecule 3's phosphate backbone are poorly constrained, and

394 Molecule 1 or 2 should instead be considered as the most accurate representation of 395 the structure. Anisotropy also prevented identification of water molecules around 396 Molecule 3. Furthermore, the $\mathrm{mF}_{\mathrm{o}}-\mathrm{DF}_{\mathrm{c}}$ map revealed several globular patches of 397 positive density in the major and minor grooves of all molecules, 3.5-4 $\AA$ away from the 398 nearest nucleic acid atom. Because these patches bore no recognizable geometric

399 features, attempts to model them with buffer components failed to improve $R_{\text {free, }}$, so they 400 were left unmodeled. Any of the mentioned issues may contribute to the high $\mathrm{R}_{\text {free }}$ 401 value.

Beyond the anisotropy, the overall high B-factors in this structure produce $2 \mathrm{mF}_{\mathrm{o}^{-}}$

403 DF $_{c}$ density that is "blurred" [53]. To enhance high-resolution features of the map for 404 visual inspection and figure preparation, Coot's Map Sharpening tool was used. B-factor 
405 adjustments used for sharpening are reported in the figure legend. Sharpening only

406 effectively revealed high-resolution features for Molecule 1 or 2, as density from

407 Molecule 3 is too anisotropically distorted.

408

409 Structure analysis and figure preparation

410 Structural model and map figures were prepared in PyMOL. Alignments were performed

411 using PyMOL's "align” function without outlier rejection. Regularized A-form and B-form

412 DNA:DNA duplexes were prepared using X3DNA's "fiber" program (options -1 and -4 ,

413 respectively), using the same sequence present in the helical portion of the

414 crystallization construct (except RNA was modeled as the corresponding DNA

415 sequence). While the A-form DNA:DNA helix may not perfectly represent a regularized

416 version of the RNA:DNA helix with our sequence $[19,20]$, "fiber" does not permit

417 generation of RNA:DNA helices with generic sequence, and the general geometric

418 features of A-form DNA:DNA vs. A-form RNA:DNA are expected to be similar enough to

419 support the conclusions drawn in this work. Base step and nucleotide geometric

420 parameters were calculated using the "find_pair" and "analyze" programs within X3DNA.

421 On graphs of these parameters, dashed lines indicating the expected value for A-form

422 or B-form DNA were calculated by performing an equivalent analysis on the X3DNA-

423 generated regularized A-form/B-form helices and taking the average across all base

424 steps/nucleotides, unless indicated otherwise. Nucleotides with A/B character exhibit a

425 spread of values around those indicated by the dashed lines (as represented more

426 accurately by the dashed ellipses in Fig 4B), and the dashed lines are drawn merely to

427 guide the reader's eye to general trends. Angles between the helical axes of the 
428 DNA:DNA and RNA:DNA duplex were calculated as the angle between the helical axis

429 vectors of the aligned regularized A-form and B-form helices. Graphs were prepared

430 using matplotlib v3.3.2 [54]. Final figures were prepared in Adobe Illustrator v25.4.1.

431

432 Acknowledgements

433 We thank J.M. Holton and J.H. Cate for data processing advice. We thank G. Meigs for

434 technical assistance at the beamline. We thank J. Kuriyan for scientific advice.

435

436 References

437 1. Broadwater DWB, Cook AW, Kim HD. First passage time study of DNA strand

438 displacement. Biophys J. 2021;120: 2400-2412. doi:10.1016/j.bpj.2021.01.043

439 2. Hays FA, Watson J, Ho PS. Caution! DNA crossing: crystal structures of Holliday

$440 \quad$ junctions. J Biol Chem. 2003;278: 49663-49666. doi:10.1074/jbc.R300033200

441 3. Kowalczykowski SC. Biochemistry of genetic recombination: energetics and

442 mechanism of DNA strand exchange. Annu Rev Biophys Biophys Chem. 1991;20:

443 539-575. doi:10.1146/annurev.bb.20.060191.002543

444 4. McKinney SA, Déclais A-C, Lilley DMJ, Ha T. Structural dynamics of individual

$445 \quad$ Holliday junctions. Nat Struct Biol. 2003;10: 93-97. doi:10.1038/nsb883

446 5. Ortiz-Lombardía M, González A, Eritja R, Aymamí J, Azorín F, Coll M. Crystal

447 structure of a DNA Holliday junction. Nat Struct Biol. 1999;6: 913-917.

448 doi:10.1038/13277 
449 6. Seeman NC, Kallenbach NR. DNA branched junctions. Annu Rev Biophys Biomol

$450 \quad$ Struct. 1994;23: 53-86. doi:10.1146/annurev.bb.23.060194.000413

451 7. Simmel FC, Yurke B, Singh HR. Principles and Applications of Nucleic Acid Strand

452 Displacement Reactions. Chem Rev. 2019;119: 6326-6369.

453 doi:10.1021/acs.chemrev.8b00580

454 8. Srinivas N, Ouldridge TE, Sulc P, Schaeffer JM, Yurke B, Louis AA, et al. On the

455 biophysics and kinetics of toehold-mediated DNA strand displacement. Nucleic

$456 \quad$ Acids Res. 2013;41: 10641-10658. doi:10.1093/nar/gkt801

457 9. Crossley MP, Bocek M, Cimprich KA. R-Loops as Cellular Regulators and Genomic Threats. Mol Cell. 2019;73: 398-411. doi:10.1016/j.molcel.2019.01.024

10. Altae-Tran H, Kannan S, Demircioglu FE, Oshiro R, Nety SP, McKay LJ, et al. The widespread IS200/IS605 transposon family encodes diverse programmable RNAguided endonucleases. Science. 2021;374: 57-65. doi:10.1126/science.abj6856

11. Karvelis T, Druteika G, Bigelyte G, Budre K, Zedaveinyte R, Silanskas A, et al. Transposon-associated TnpB is a programmable RNA-guided DNA endonuclease. Nature. 2021;599: 692-696. doi:10.1038/s41586-021-04058-1

12. Chen JS, Doudna JA. The chemistry of Cas 9 and its CRISPR colleagues. Nat Rev 
469

470

471

472

473

474

475

476

477

478

479

480

481

482

483

484

485

486

487

488

489

14. Pickar-Oliver A, Gersbach CA. The next generation of CRISPR-Cas technologies and applications. Nat Rev Mol Cell Biol. 2019;20: 490-507. doi:10.1038/s41580019-0131-5

15. Sternberg SH, Redding S, Jinek M, Greene EC, Doudna JA. DNA interrogation by the CRISPR RNA-guided endonuclease Cas9. Nature. 2014;507: 62-67. doi:10.1038/nature13011

16. Cofsky JC, Karandur D, Huang CJ, Witte IP, Kuriyan J, Doudna JA. CRISPRCas12a exploits R-loop asymmetry to form double-strand breaks. Wolberger C, Bailey S, Ke A, White MF, editors. eLife. 2020;9: e55143. doi:10.7554/eLife.55143

17. Aymami J, Coll M, Marel GA van der, Boom JH van, Wang AH, Rich A. Molecular structure of nicked DNA: a substrate for DNA repair enzymes. PNAS. 1990;87: 2526-2530.

18. Roll C, Ketterlé C, Faibis V, Fazakerley GV, Boulard Y. Conformations of nicked and gapped DNA structures by NMR and molecular dynamic simulations in water. Biochemistry. 1998;37: 4059-4070. doi:10.1021/bi972377w

19. Fedoroff OYu null, Salazar M, Reid BR. Structure of a DNA:RNA hybrid duplex. Why RNase H does not cleave pure RNA. J Mol Biol. 1993;233: 509-523. doi:10.1006/jmbi.1993.1528

20. Horton NC, Finzel BC. The Structure of an RNA/DNA Hybrid: A Substrate of the Ribonuclease Activity of HIV-1 Reverse Transcriptase. Journal of Molecular Biology. 1996;264: 521-533. doi:10.1006/jmbi.1996.0658 
490 21. Milman G, Langridge R, Chamberlin MJ. The structure of a DNA-RNA hybrid. Proc

$491 \quad$ Natl Acad Sci U S A. 1967;57: 1804-1810.

492 22. Zhu L, Salazar M, Reid BR. DNA duplexes flanked by hybrid duplexes: the solution 493 structure of chimeric junctions in [r(cgcg)d(TATACGCG)]2. Biochemistry. 1995;34:

494 2372-2380. doi:10.1021/bi00007a033

23. Dickerson RE, Goodsell DS, Neidle S. ...the tyranny of the lattice... Proc Natl Acad Sci U S A. 1994;91: 3579-3583. doi:10.1073/pnas.91.9.3579

24. Drew HR, Wing RM, Takano T, Broka C, Tanaka S, Itakura K, et al. Structure of a B-DNA dodecamer: conformation and dynamics. Proc Natl Acad Sci U S A. 1981;78: 2179-2183. doi:10.1073/pnas.78.4.2179 Media; 1984.

26. El Hassan MA, Calladine CR. Conformational characteristics of DNA: empirical classifications and a hypothesis for the conformational behaviour of dinucleotide steps. Philosophical Transactions of the Royal Society of London Series A: Mathematical, Physical and Engineering Sciences. 1997;355: 43-100. structures. J Mol Biol. 2000;300: 819-840. doi:10.1006/jmbi.2000.3690 
509

510

511

512

513

514

515

516

517

518

519

520

521

522

523

524

525

526

527

528

529

28. Ban C, Ramakrishnan B, Sundaralingam M. A single 2'-hydroxyl group converts BDNA to A-DNA. Crystal structure of the DNA-RNA chimeric decamer duplex $d(C C G G C) r(G) d(C C G G)$ with a novel intermolecular $G-C$ base-paired quadruplet. J Mol Biol. 1994;236: 275-285. doi:10.1006/jmbi.1994.1134

29. Egli M, Usman N, Zhang SG, Rich A. Crystal structure of an Okazaki fragment at 2A resolution. Proc Natl Acad Sci U S A. 1992;89: 534-538. doi:10.1073/pnas.89.2.534

30. Egli M, Usman N, Rich A. Conformational influence of the ribose 2'-hydroxyl group: crystal structures of DNA-RNA chimeric duplexes. Biochemistry. 1993;32: 32213237.

31. Wahl MC, Sundaralingam M. B-form to A-form conversion by a 3'-terminal ribose: crystal structure of the chimera d(CCACTAGTG)r(G). Nucleic Acids Res. 2000;28: 4356-4363. doi:10.1093/nar/28.21.4356

32. Wang AH, Fujii S, van Boom JH, van der Marel GA, van Boeckel SA, Rich A. Molecular structure of r(GCG)d(TATACGC): a DNA--RNA hybrid helix joined to double helical DNA. Nature. 1982;299: 601-604. doi:10.1038/299601a0

33. Mellema JR, Haasnoot CA, van der Marel GA, Wille G, van Boeckel CA, van Boom $\mathrm{JH}$, et al. Proton NMR studies on the covalently linked RNA-DNA hybrid $r(G C G) d(T A T A C G C)$. Assignment of proton resonances by application of the nuclear Overhauser effect. Nucleic Acids Res. 1983;11: 5717-5738. doi:10.1093/nar/11.16.5717 
530 34. Salazar M, Fedoroff OYu null, Zhu L, Reid BR. The solution structure of the $531 \quad \mathrm{r}(\mathrm{gcg}) \mathrm{d}(\mathrm{TATACCC}): \mathrm{d}(\mathrm{GGGTATACGC})$ Okazaki fragment contains two distinct

532 duplex morphologies connected by a junction. J Mol Biol. 1994;241: 440-455.

533 doi:10.1006/jmbi.1994.1519

534 35. Salazar M, Fedoroff OY, Reid BR. Structure of chimeric duplex junctions: solution

535 conformation of the retroviral Okazaki-like fragment

536 r(ccca)d(AATGA).d(TCATTTGGG) from Moloney murine leukemia virus. Biochemistry. 1996;35: 8126-8135. doi:10.1021/bi9528917

36. Selsing E, Wells RD, Early TA, Kearns DR. Two contiguous conformations in a nucleic acid duplex. Nature. 1978;275: 249-250. doi:10.1038/275249a0

37. Häse F, Zacharias M. Free energy analysis and mechanism of base pair stacking in nicked DNA. Nucleic Acids Res. 2016;44: 7100-7108. doi:10.1093/nar/gkw607

38. Pacesa M, Jinek M. Mechanism of R-loop formation and conformational activation of Cas9. 2021. p. 2021.09.16.460614. Available: https://www.biorxiv.org/content/10.1101/2021.09.16.460614v1

39. Zhu X, Clarke R, Puppala AK, Chittori S, Merk A, Merrill BJ, et al. Cryo-EM structures reveal coordinated domain motions that govern DNA cleavage by Cas9. Nat Struct Mol Biol. 2019;26: 679-685. doi:10.1038/s41594-019-0258-2 
551 41. Lapinaite A, Knott GJ, Palumbo CM, Lin-Shiao E, Richter MF, Zhao KT, et al. DNA capture by a CRISPR-Cas9-guided adenine base editor. Science. 2020;369: 566571. doi:10.1126/science.abb1390

42. Cavaluzzi MJ, Borer PN. Revised UV extinction coefficients for nucleoside-5'monophosphates and unpaired DNA and RNA. Nucleic Acids Res. 2004;32: e13. doi:10.1093/nar/gnh015

43. Kabsch W. XDS. Acta Cryst D. 2010;66: 125-132.

44. Kabsch W. Integration, scaling, space-group assignment and post-refinement. Acta Cryst D. 2010;66: 133-144. doi:10.1107/S0907444909047374

45. Tickle IJ, Flensburg C, Keller P, Paciorek W, Sharff A, Vonrhein C, et al. STARANISO. Cambridge, United Kingdom: Global Phasing Ltd.; 2018. Available: http://staraniso.globalphasing.org/cgi-bin/staraniso.cgi

46. McCoy AJ, Grosse-Kunstleve RW, Adams PD, Winn MD, Storoni LC, Read RJ. Phaser crystallographic software. J Appl Cryst. 2007;40: 658-674. doi:10.1107/S0021889807021206

47. Liebschner D, Afonine PV, Baker ML, Bunkóczi G, Chen VB, Croll TI, et al. Macromolecular structure determination using X-rays, neutrons and electrons:

569 recent developments in Phenix. Acta Cryst D. 2019;75: 861-877. doi:10.1107/S2059798319011471 
571 48. Lu X-J, Olson WK. 3DNA: a software package for the analysis, rebuilding and

572 visualization of three-dimensional nucleic acid structures. Nucleic Acids Res.

$573 \quad$ 2003;31: 5108-5121. doi:10.1093/nar/gkg680

574 49. Emsley P, Lohkamp B, Scott WG, Cowtan K. Features and development of Coot.

575 Acta Crystallogr D Biol Crystallogr. 2010;66: 486-501.

576 doi:10.1107/S0907444910007493

577 50. Painter J, Merritt EA. TLSMD web server for the generation of multi-group TLS

578 models. J Appl Cryst. 2006;39: 109-111. doi:10.1107/S0021889805038987

579 51. Painter J, Merritt EA. Optimal description of a protein structure in terms of multiple

580 groups undergoing TLS motion. Acta Cryst D. 2006;62: 439-450.

581 doi:10.1107/S0907444906005270

52. Kleywegt GJ, Brünger AT. Checking your imagination: applications of the free R value. Structure. 1996;4: 897-904. doi:10.1016/s0969-2126(96)00097-4

53. Liu C, Xiong Y. Electron density sharpening as a general technique in crystallographic studies. J Mol Biol. 2014;426: 980-993. doi:10.1016/j.jmb.2013.11.014

54. Hunter JD. Matplotlib: A 2D Graphics Environment. Computing in Science Engineering. 2007;9: 90-95. doi:10.1109/MCSE.2007.55 
591 S1 Fig. Additional geometric details of the A-to-B transition.

592 (A) X-displacement of the 11 base steps of the 12-bp helix. Black, DNA; red, RNA. (B)

593 Inclination of the 11 base steps of the 12-bp helix. (C) Slide of the 11 base steps of the

594 12-bp helix. (D) Pseudorotation phase angles for the ribose/deoxyribose conformation

595 at every nucleotide within the 12-bp helix (24 data points per molecule). The modeled

596 sugar conformations might not be unique solutions for this dataset, as in many cases

597 these structural details cannot be directly discerned from the $2 \mathrm{mF}_{\mathrm{o}}-\mathrm{DF} \mathrm{F}_{\mathrm{c}}$ map. For this

598 dataset, the most reliable parameters are those defined directly by the nucleobase and

599 phosphate positions, which appear clearly in the $2 \mathrm{mF}_{\mathrm{o}}-\mathrm{DF}_{\mathrm{c}}$ map (and likely impose

600 indirect geometric constraints on the sugar pucker).

601 S2 Fig.

602

603 S2 Fig. Nucleotide torsion angles for Molecules 2 and 3.

604 Analogous to Fig 4B. 
A

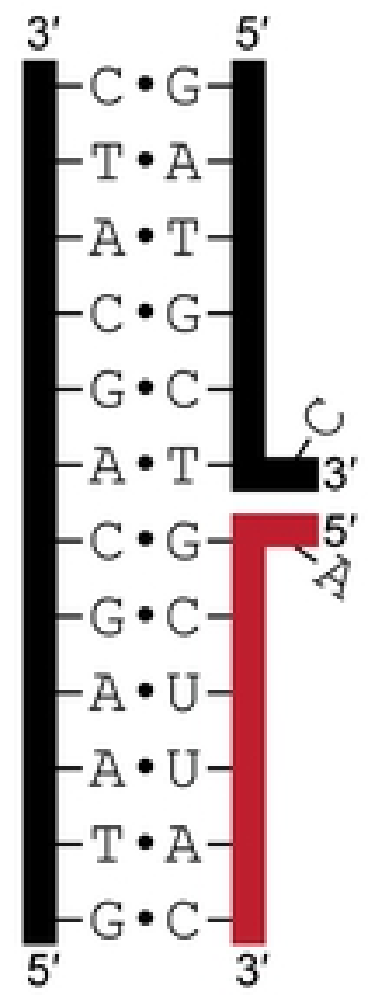

B
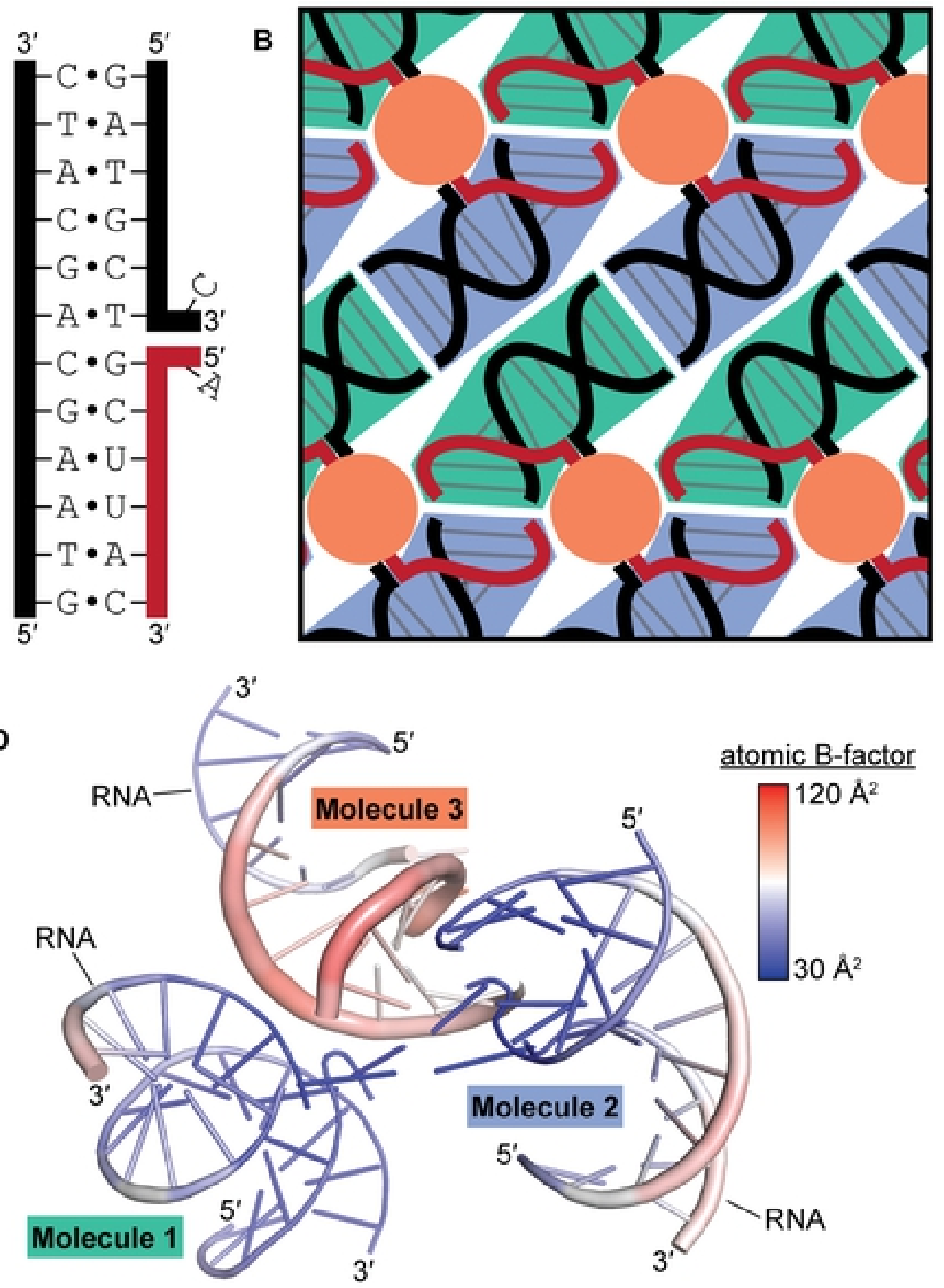
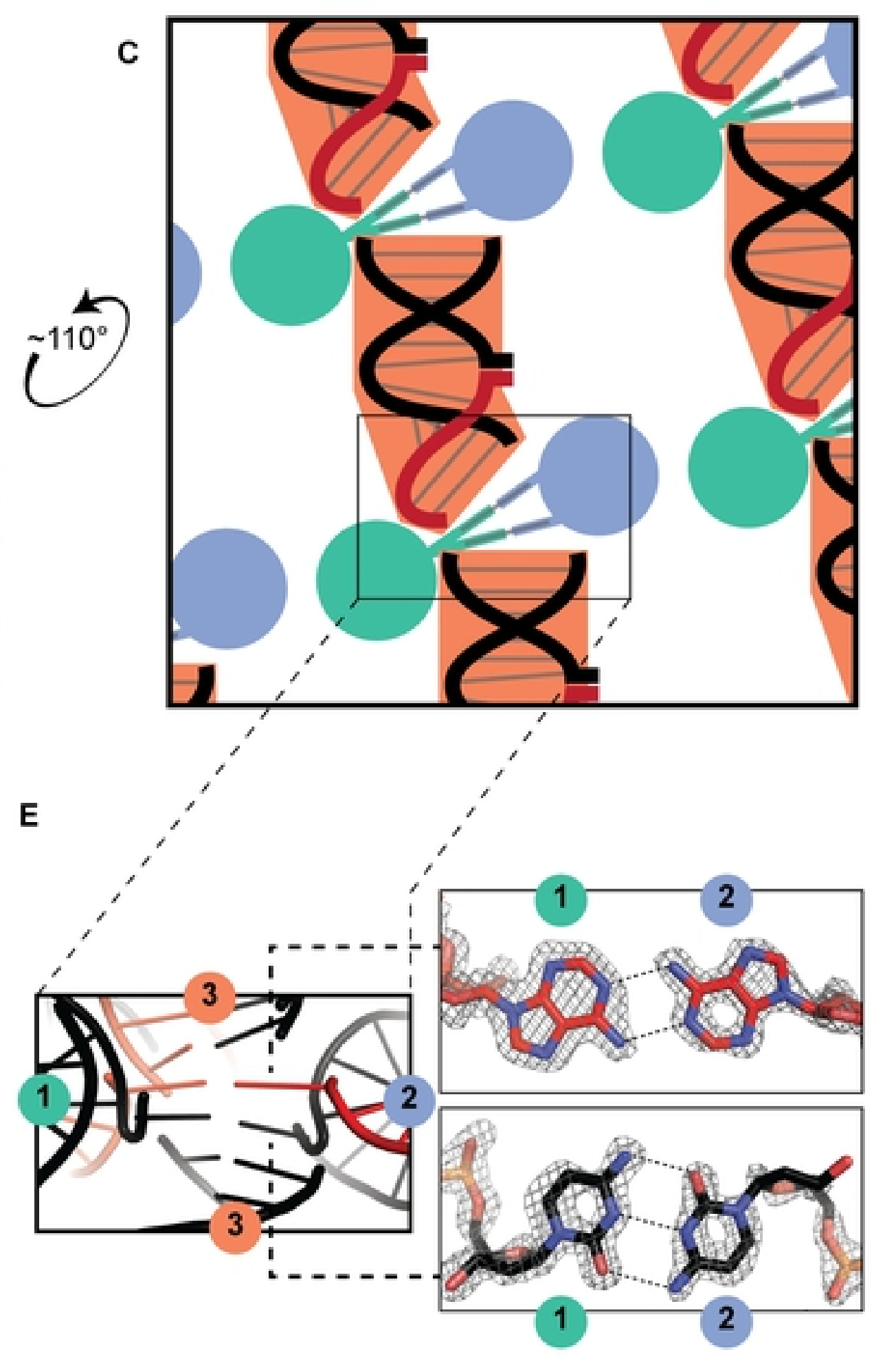

Fig 1 

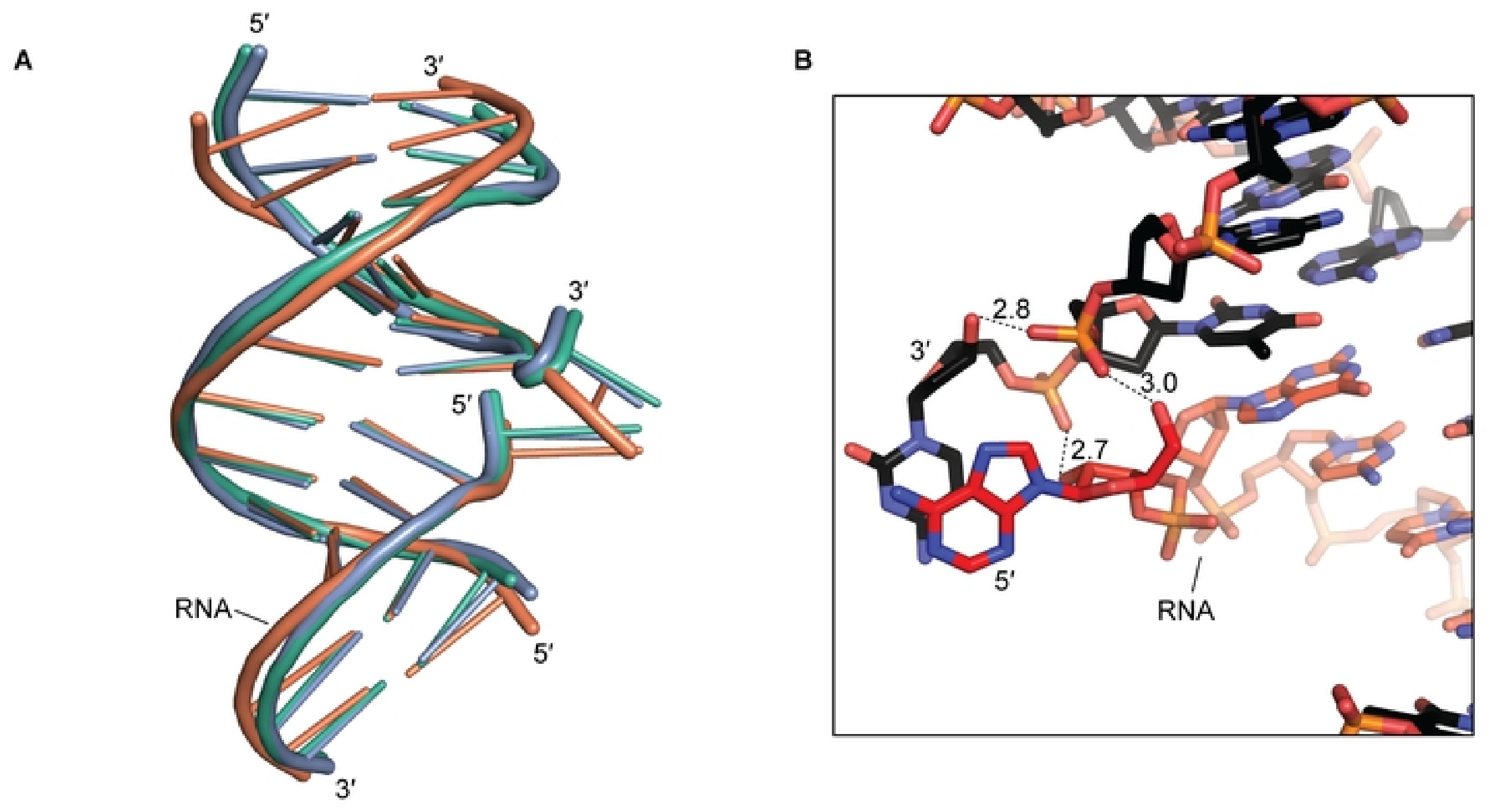

Fig 2 

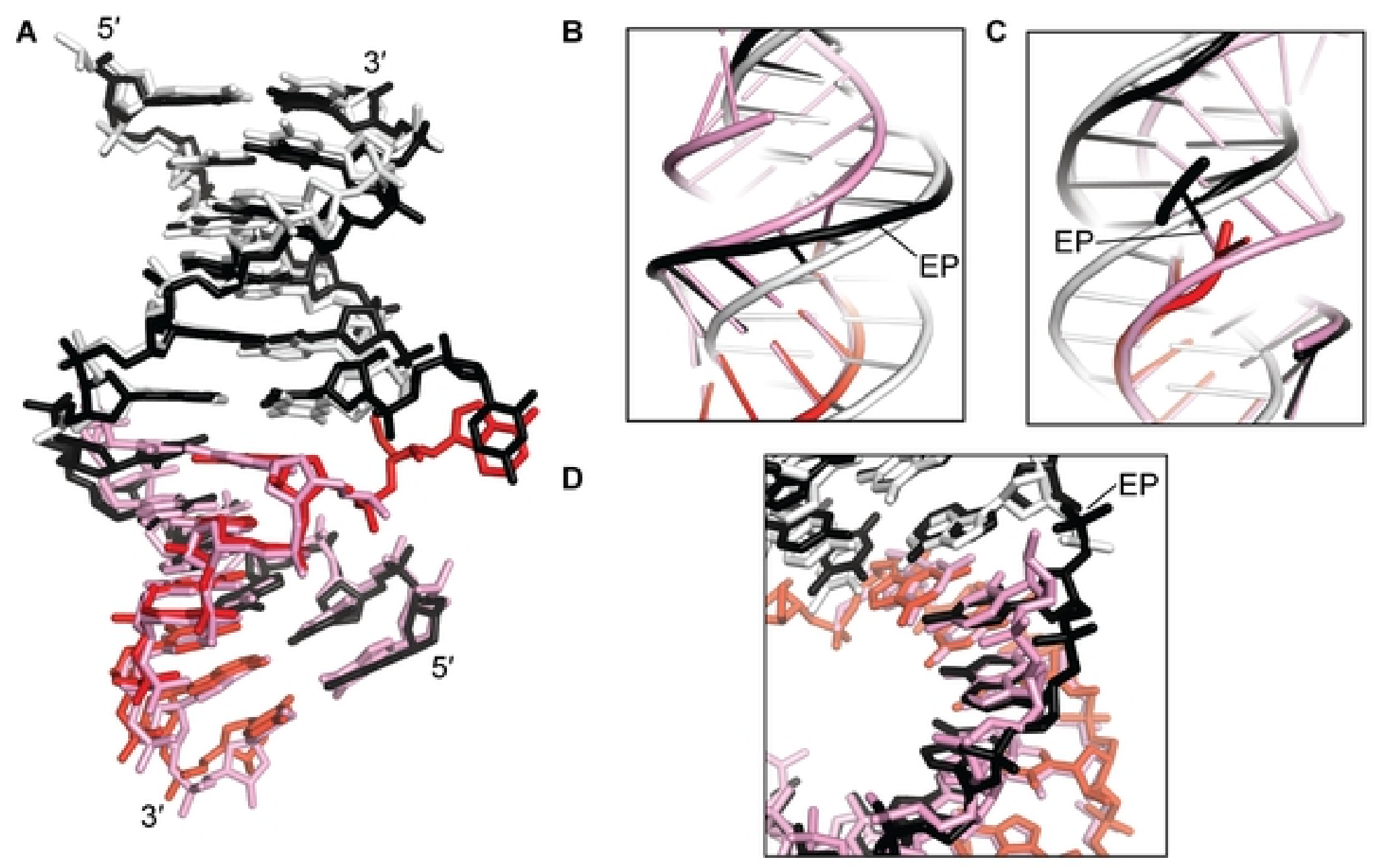

Fig 3 
A
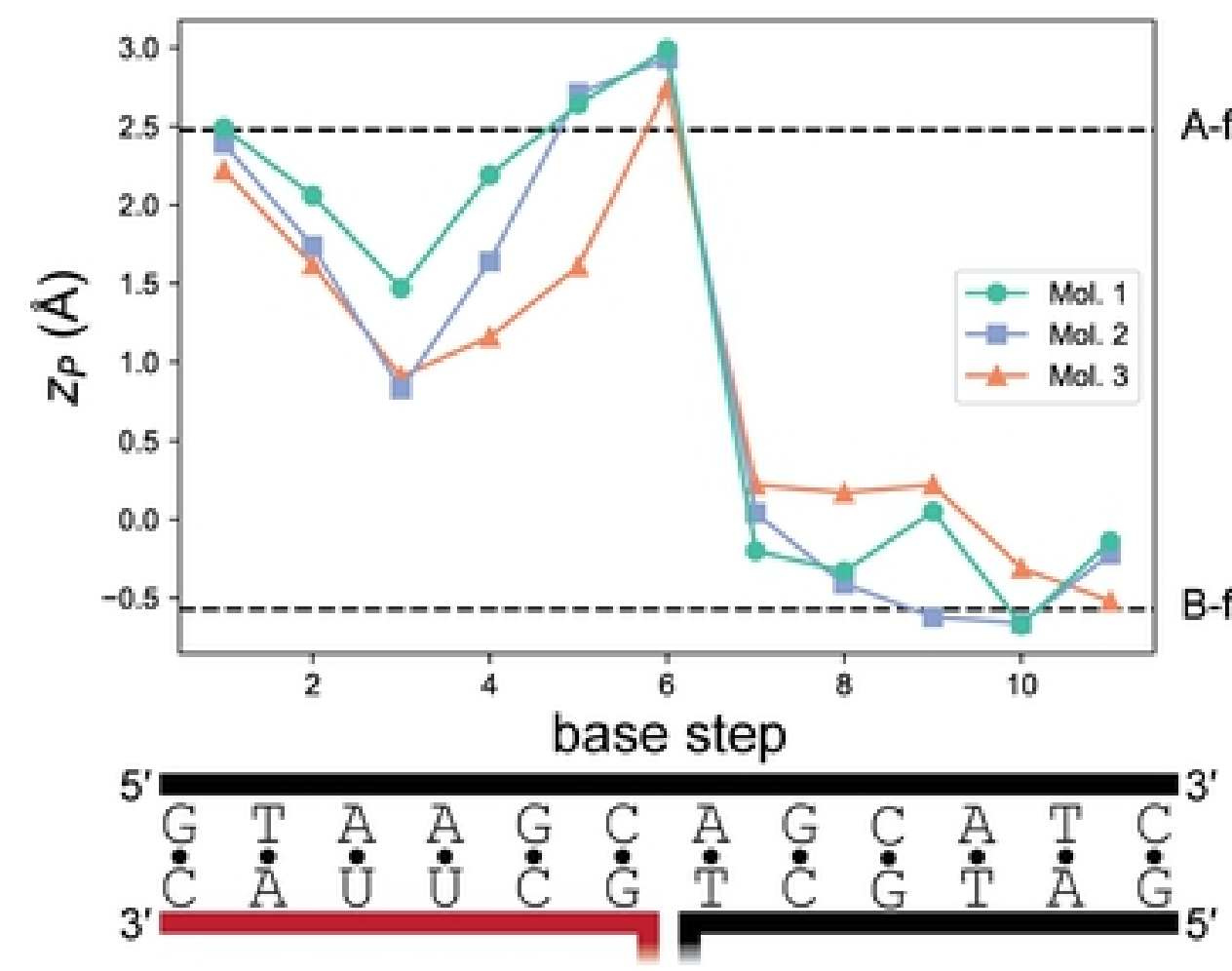

B

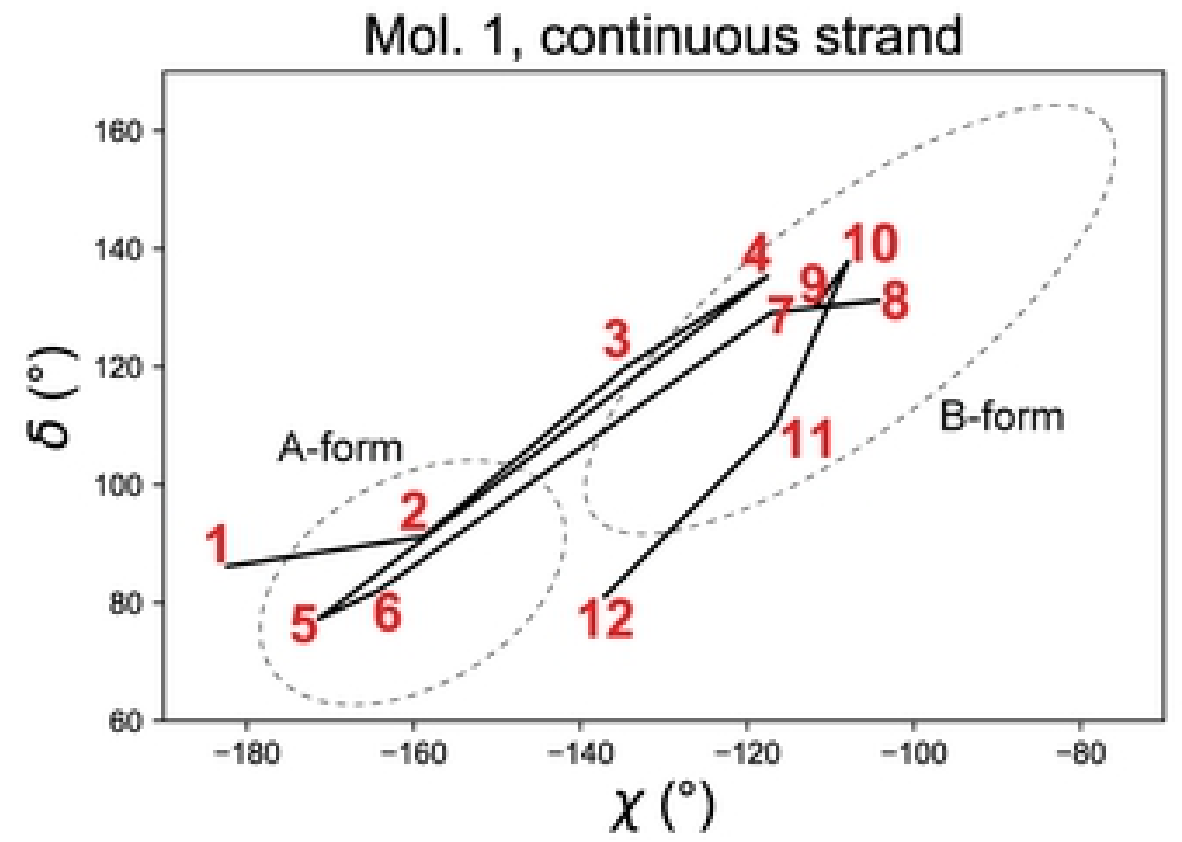

C
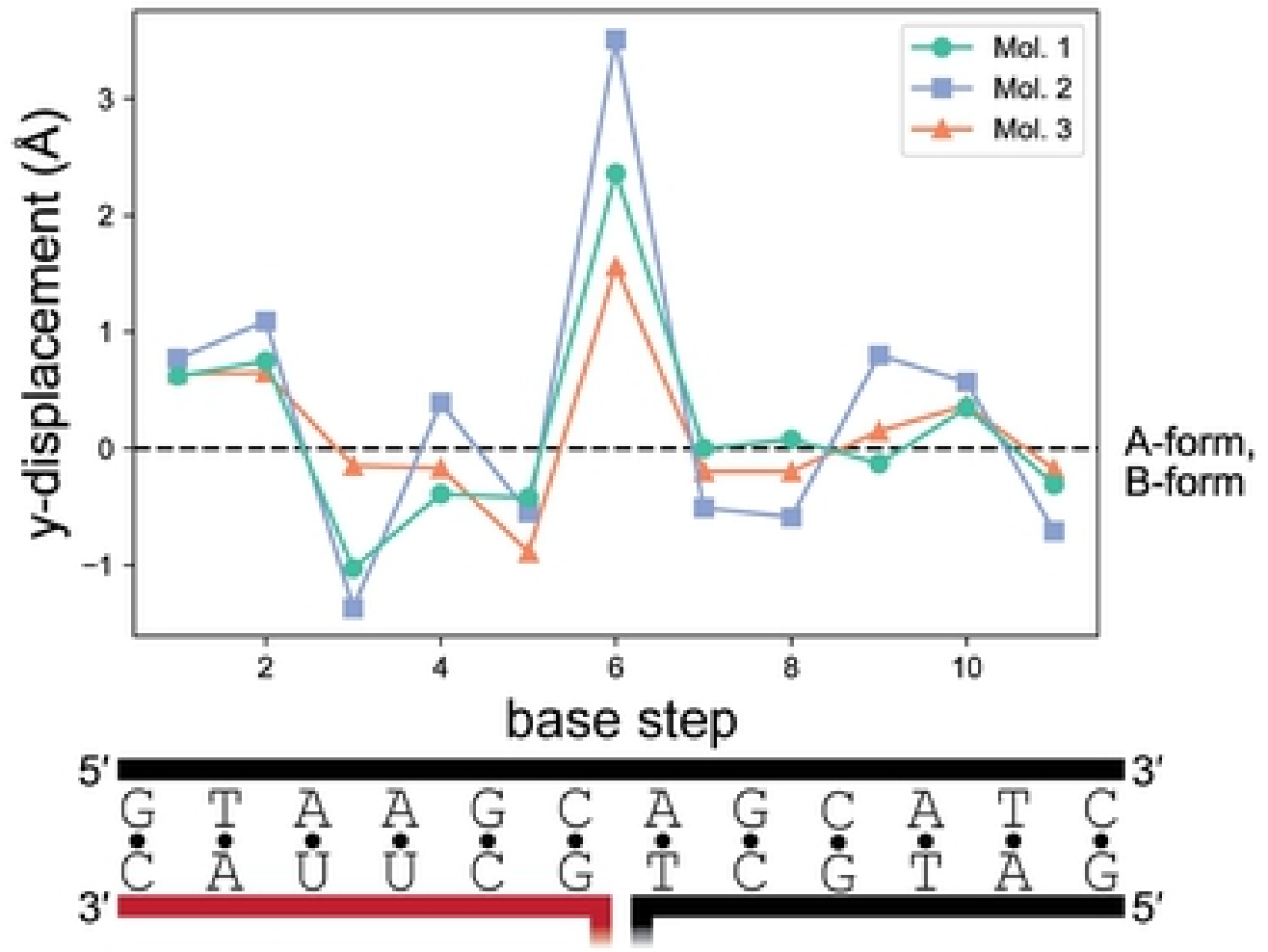

Mol. 1, discontinuous strand

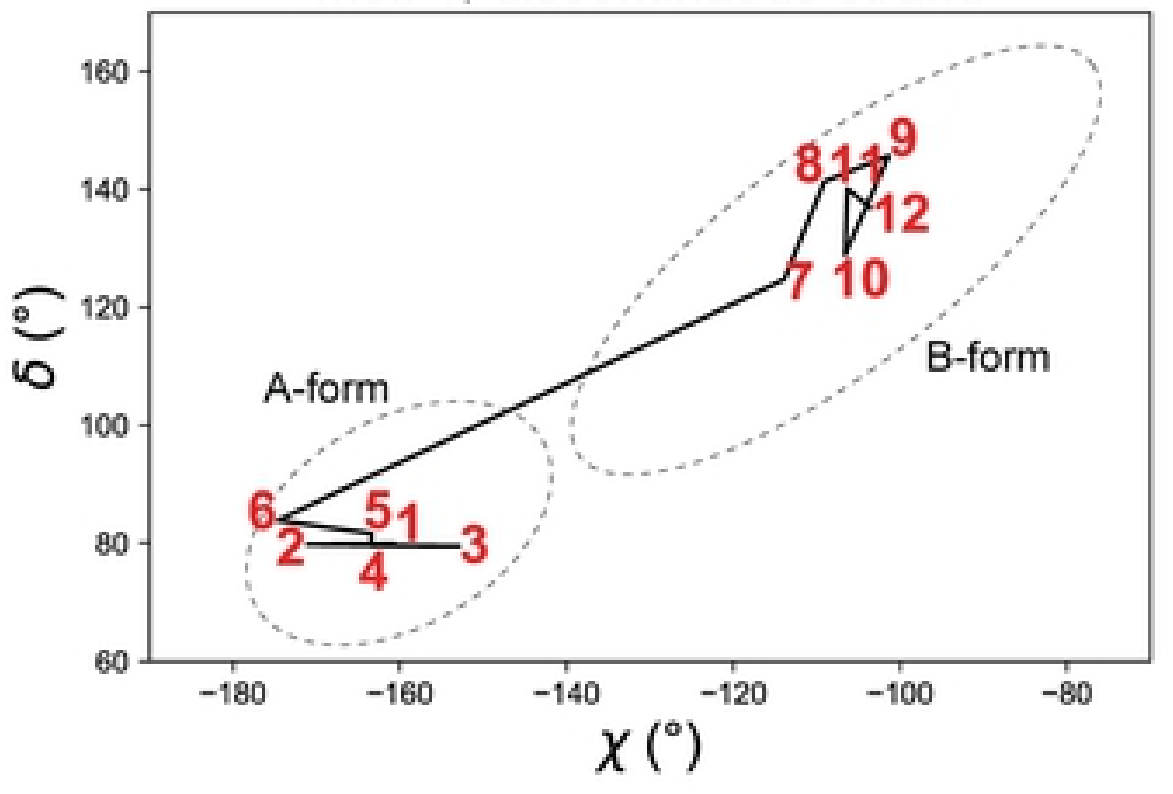

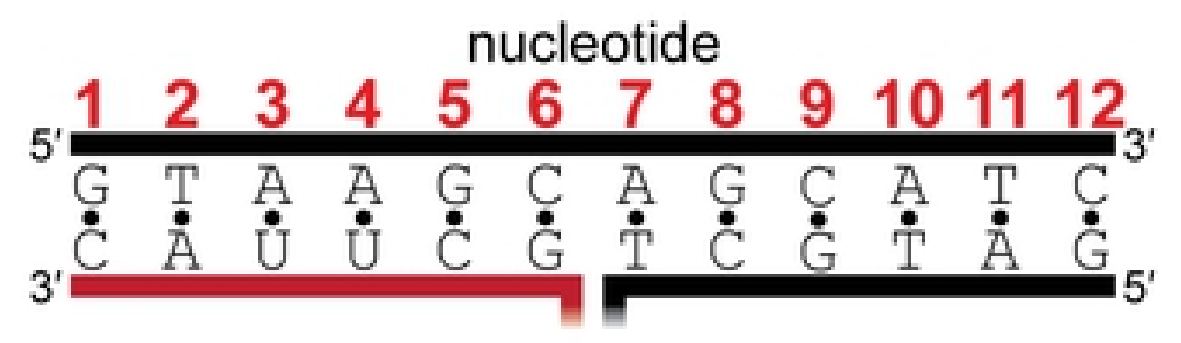

Fig 4 


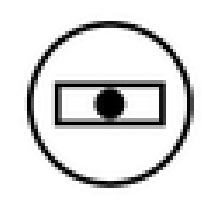

DNA:DNA
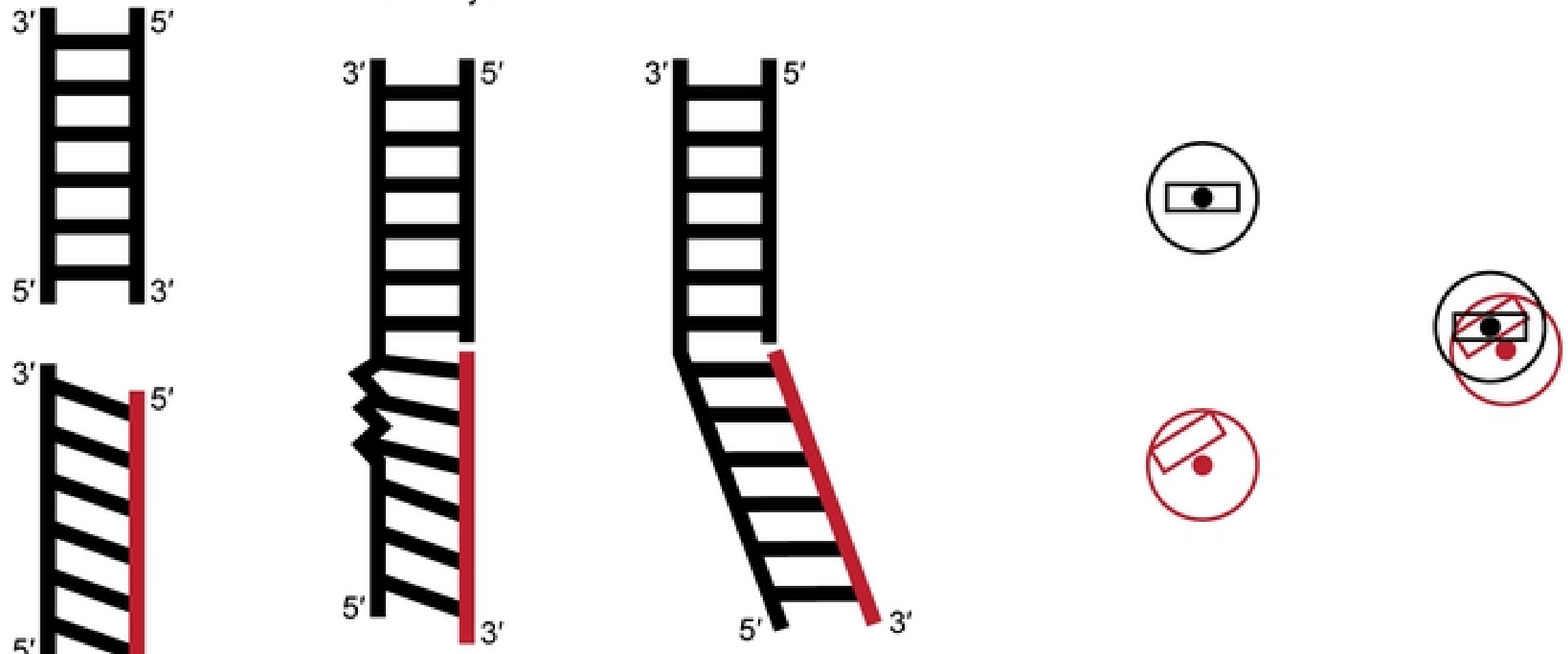

RNA:DNA
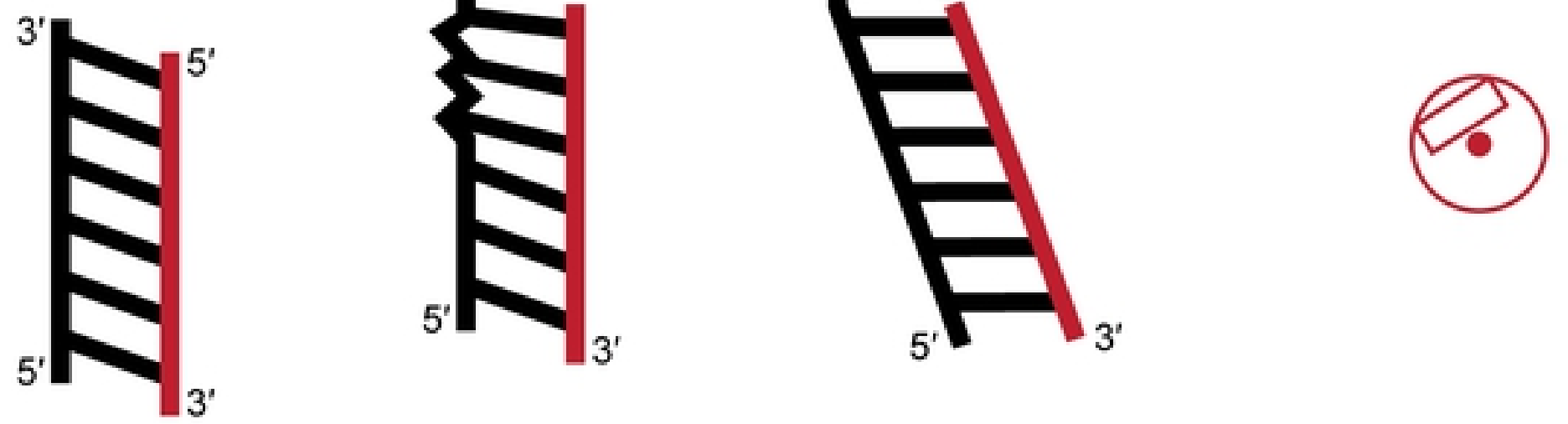

(A-form)

Fig 5 